\title{
Relação entre valores pessoais, social connectedness e desenvolvimento da identidade em adultos emergentes portugueses
}

The Relationship between Personal Values, Social Connectedness and Identity Development of Portuguese Emerging Adults Rapport entre les valeurs personnelles, le social connectedness et le développement de l'identité chez les adultes portugais émergents

Ana Prioste, Cristina Ascensão, Eunice Magalhães e Inês Jongenelen

\section{OpenEdition}

\section{Journals}

Edição electrónica

URL: http://journals.openedition.org/rccs/8522

DOI: $10.4000 /$ rccs.8522

ISSN: 2182-7435

\section{Editora}

Centro de Estudos Sociais da Universidade de Coimbra

Edição impressa

Data de publição: 1 maio 2019

Paginação: 79-100

ISSN: 0254-1106

Refêrencia eletrónica

Ana Prioste, Cristina Ascensão, Eunice Magalhães e Inês Jongenelen, « Relação entre valores pessoais, social connectedness e desenvolvimento da identidade em adultos emergentes portugueses ", Revista Crítica de Ciências Sociais [Online], 118 | 2019, posto online no dia 26 abril 2019, consultado o 19 março 2020. URL : http://journals.openedition.org/rccs/8522 ; DOI : https://doi.org/10.4000/ rccs. 8522 


\title{
ANA PRIOSTE, CRISTINA ASCENSÃO, EUNICE MAGALHÃES, INÊS JONGENELEN
}

\section{Relação entre valores pessoais, social connectedness e desenvolvimento da identidade em adultos emergentes portugueses}

\begin{abstract}
Os valores pessoais e o social connectedness contribuem para o desenvolvimento identitário. Através de um desenho quantitativo transversal e de uma amostra de 275 adultos emergentes, o presente estudo pretendeu analisar: a relação entre os valores pessoais coletivistas e individualistas, os dois ciclos de desenvolvimento da identidade (formação e avaliação do compromisso) e o social connectedness; se os valores pessoais são preditores dos ciclos de desenvolvimento da identidade; e o papel mediador do social connectedness na relação entre os valores pessoais e os ciclos de desenvolvimento da identidade. Os resultados mostram que o social connectedness tem um papel de mediador da relação entre os valores pessoais e os ciclos de desenvolvimento da identidade, sugerindo que o sentimento de proximidade interpessoal funcione como um mecanismo de confirmação em relação aos valores pessoais e que contribua para o desenvolvimento identitário. Este estudo tem implicações para a literatura relacionada com o desenvolvimento da identidade na adultez emergente.
\end{abstract}

Palavras-chave: adultos emergentes; desenvolvimento individual; identidade pessoal; interação social; Portugal.

Os valores, enquanto "conceitos ou crenças acerca de comportamentos ou estados desejados que guiam e avaliam o comportamento e os acontecimentos" (Schwartz e Bilsky, 1990: 878), têm um papel relevante na preservação da identidade pessoal, dando coerência e continuidade ao comportamento (Caprara et al., 2006). A importância atribuída a cada valor pessoal é, simultaneamente, um reflexo e um refletor da significação das experiências, da personalidade, do contexto e da cultura em que a pessoa se insere (Hermans e Oles, 1993; Prince-Gibson e Schwartz, 1998; Prioste et al., 2012). Da mesma forma, a identidade, sendo composta por valores, crenças e ideias, é influenciada por objetivos pessoais e normas sociais (Bardi et al., 2014). 
A literatura tem realçado o papel dos valores pessoais na regulação das interações sociais (e.g., a importância do valor "honestidade" na relação entre pares) e a importância das relações sociais no desenvolvimento da identidade (Lee e Robbins, 1995; Schwartz et al., 2006). Desta forma, este trabalho pretendeu estudar: a relação entre os valores pessoais individualistas e coletivistas, os ciclos de identidade e o social connectedness $;{ }^{1} \mathrm{o}$ impacto dos valores pessoais nos ciclos de desenvolvimento da identidade propostos por Luyckx et al. (2006); e o papel do social connectedness enquanto mediador da relação entre os valores pessoais e os ciclos de desenvolvimento da identidade. Deste modo, procurou-se contribuir para o enriquecimento e para o aprofundamento do conhecimento científico sobre o impacto de variáveis individuais e relacionais no desenvolvimento da identidade em adultos emergentes portugueses.

A relevância do estudo deste tema pode ser fundamentada por vários pontos. Diversos autores têm sugerido uma associação entre os valores pessoais e os processos de desenvolvimento da identidade, apontando para a relevância do compromisso com valores pessoais no desenvolvimento identitário (Bardi et al., 2014; Duriez et al., 2012; Hitlin, 2003). Por exemplo, os valores influenciam os objetivos pessoais e a escolha dos grupos de pertença, o que se repercute, necessariamente, nas situações experienciadas e no comportamento individual (Seligman e Katz, 1996). Duriez et al. (2012) notaram que os objetivos e valores, enquanto conteúdos da identidade, e o processo de formação identitária se influenciem reciprocamente. Contudo, apesar de a literatura relevar a relação entre os valores pessoais e a identidade, a investigação nesta área tem sido escassa e não foram identificados estudos que analisem a relação entre os valores pessoais e os processos e ciclos de desenvolvimento da identidade propostos por Luyckx et al. (2006). Do mesmo modo, o trabalho de Davidson e Youniss (1991) sugere a relevância do papel das relações com o grupo de pares e das comparações sociais no desenvolvimento identitário, no sentido de permitirem a consolidação das crenças sobre o/a próprio/a e os outros. Não obstante, e apesar de existirem estudos que relacionam as relações sociais (e.g., Adams e Marshall, 1996; Cicognani et al., 2014; Davidson e Youniss, 1991) e a identidade, não foram encontrados estudos que relacionem o social connectedness e os processos e ciclos de desenvolvimento da identidade propostos por Luyckx et al. (2006).

\footnotetext{
${ }^{1}$ Tendo em conta que não existe uma tradução fidedigna da expressão social connectedness, no presente estudo manter-se-á a expressão original que designa o sentido de pertença e de proximidade com o mundo social (Lee e Robbins, 1995, 1998).
} 
$\mathrm{Na}$ adultez emergente, o processo de desenvolvimento da identidade ocorre através da exploração das opções disponíveis, especialmente nas áreas relacionais e laborais, permitindo a clarificação do sentido identitário (i.e., quem sou?) e dos planos para a vida futura (i.e., o que quero fazer na vida?) (Arnett, 2015). Deste modo, o desenvolvimento da identidade, bem como a vivência da instabilidade, a experienciação de possibilidades/ /otimismo, o sentimento de ambiguidade e a autocentração, são tarefas desenvolvimentais centrais desta etapa (Arnett, 2000, 2015). Considerando que o desenvolvimento da identidade tem um impacto no funcionamento e no bem-estar individual (Erikson, 1968), a área da psicologia clínica poderá beneficiar com o aprofundamento da compreensão deste tópico para desenvolver intervenções centradas nos processos identitários em adultos emergentes, através do foco nos valores pessoais e no social connectedness. Para além disso, importa considerar a relevância que, por um lado, os valores coletivistas e o social connectedness poderão ter para a exploração da identidade na área relacional e, por outro, que os valores individualistas poderão ter para a exploração da identidade na área laboral, nomeadamente, através da realização e do equilíbrio pessoal.

\section{Desenvolvimento da identidade: modelos explicativos}

A teoria do desenvolvimento psicossocial (Erikson, 1968) serviu de base ao desenvolvimento de vários modelos teóricos explicativos do desenvolvimento da identidade, nomeadamente, o modelo dos estados identitários (Marcia, 2002), o modelo do processo (Grotevant, 1987) e o modelo integrativo do desenvolvimento identitário (Luyckx et al., 2008). O modelo dos estados identitários de Marcia (2002) foi desenvolvido a partir da validação empírica do trabalho de Erikson (1968) e baseia-se na interseção de dois processos centrais na formação da identidade: o compromisso - que remete para a adesão a um conjunto de objetivos, valores e crenças - e a exploração, i.e., o questionamento ativo das alternativas identitárias. A partir destes processos, Marcia (2002) definiu quatro estatutos de identidade: identidade realizada, i.e., o estabelecimento de um compromisso após a exploração de alternativas; difusão da identidade, i.e., ausência de exploração de alternativas e de compromissos; identidade fechada, i.e., definição de compromissos e objetivos sem um período de exploração prévia; e identidade moratória, caracterizada por um processo de exploração e pela dificuldade de tomada de decisão e de estabelecimento de um compromisso.

O modelo integrativo do desenvolvimento identitário de Luyckx et al. (2008) corresponde a uma reformulação do modelo de estados identitários de Marcia (2002) e centra-se na forma como o processo de desenvolvimento 
identitário pode ser formulado após a formação de compromissos. Luyckx et al. (2008) distinguiram cinco processos de identidade - exploração em amplitude, exploração em profundidade, exploração ruminativa, compromisso e identificação com compromisso. ${ }^{2}$ Os autores consideram que o desenvolvimento da identidade ocorre através de um processo com dois ciclos consecutivos de formação da identidade - formação do compromisso e avaliação do compromisso - que integram quatro processos distintos. O primeiro ciclo - formação do compromisso - centra-se nos processos através dos quais os indivíduos exploram diferentes alternativas da identidade (exploração de amplitude ou pró-ativa) e aderem a compromissos de identidade (compromisso, i.e., adesão a valores, objetivos e crenças) (Luyckx et al., 2013). O segundo ciclo de identidade - avaliação do compromisso - foca os processos através dos quais os indivíduos reavaliam os seus compromissos de identidade (exploração em profundidade, i.e., avaliação e exploração dos compromissos atuais) e avalia o grau em que eles se identificam e se sentem seguros em relação aos seus compromissos (identificação com compromisso, i.e., grau em que os compromissos se integram no seu sentido de self) (ibidem). O processo de identidade exploração ruminativa é perspetivado como um bloqueador do desenvolvimento identitário ou um processo não adaptativo, tendo sido adicionado ao modelo posteriormente (Luyckx et al., 2008). Nas pessoas com níveis elevados de exploração ruminativa, a dificuldade em encontrar respostas satisfatórias às questões identitárias leva-as a um questionamento contínuo em relação a essas questões e a sentimentos de incerteza e incompetência (Luyckx et al., 2011).

Os processos identitários de exploração pró-ativa e de adesão a compromissos têm sido positivamente associados à satisfação de necessidades psicológicas básicas (e.g., autonomia, competência) e à autoeficácia na tomada de decisões, i.e., escolha de objetivos/metas e definição de planos para o futuro (Cordeiro et al., 2015). A literatura tem mostrado a associação entre os processos identitários de exploração e compromisso e o bem-estar, a autodescoberta e o ajustamento positivo (Ritchie et al., 2013; Schwartz et al., 2011). Por exemplo, o estudo de Karás et al. (2014) revelou uma associação positiva entre os processos de compromisso e exploração em profundidade e o bem-estar.

\footnotetext{
${ }^{2}$ De acordo com os resultados do estudo de Luyckx et al. (2008), a identidade realizada encontra-se positivamente correlacionada com os processos de compromisso, identificação com o compromisso, exploração em profundidade e exploração em amplitude; a difusão da identidade encontra-se negativamente associada a: compromisso, identificação com compromisso, exploração em profundidade e exploração em amplitude.
} 


\section{Valores pessoais}

A teoria dos valores individuais básicos de Schwartz e Bilsky (1990) propõe um agrupamento dos valores em torno de um continuum motivacional e sugere que os valores possam ser diferenciados consoante o foco individual versus coletivo (Schwartz et al., 2012). À semelhança de outros estudos realizados no contexto português (i.e., Prioste et al., 2015, 2017), no presente estudo, os valores do Schwartz Values Survey (SVS) foram diferenciados em valores coletivistas (valores de interdependência, que enfatizam a conexão com os outros) e valores individualistas, i.e., focados na independência em relação aos outros.

Alguns trabalhos têm explorado a relação entre os valores pessoais e o processo de formação da identidade. Por exemplo, o trabalho de Duriez et al. (2012), centrado na relação entre valores de abertura à mudança e de conservação ${ }^{3}$ e os estilos de identidade informativa, normativa e difusa, com uma amostra de 806 adolescentes, mostrou que: (a) os estilos de identidade informativo e difuso são preditores negativos de valores de conservação; e (b) o estilo normativo é um preditor positivo de valores de conservação. Alguns autores têm sugerido que os valores pessoais contribuem para a conexão com o mundo social (e.g., Gecas, 2000; Hitlin, 2003). Por exemplo, o trabalho de Olsson et al. (2012) revelou que os valores pró-sociais (e.g., honestidade, justiça, coragem, generosidade e bondade) estão associados positivamente ao desenvolvimento de relações positivas com os outros e com o mundo e que a adesão a estes valores influencia o social connectedness e o bem-estar.

\section{Social connectedness}

A proximidade com o mundo social desenvolve-se desde a infância, prolonga-se durante toda a vida, atingindo o exponente máximo na adolescência (Lee et al., 2001; Lee e Robbins, 1995). Lee e Robbins (1998) definiram o social connectedness como um sentimento interno de pertença e de proximidade interpessoal com vários grupos (e.g., família, amigos, colegas) que funciona como um esquema relacional ou uma estrutura cognitiva que representa os padrões de relacionamento interpessoal. O social connectedness pode ser descrito como um continuum que oscila entre níveis elevados e níveis baixos. As pessoas com elevados níveis de social connectedness tendem a sentir-se próximas dos outros, participam

\footnotetext{
3 De acordo com a teoria de Schwartz (1992), os valores de abertura à mudança englobam valores de autodeterminação, estimulação e hedonismo; os valores de conservação englobam valores de segurança, conformidade e tradição.
} 
em atividades sociais e desenvolvem um sentimento de identificação com os outros (Lee et al., 2001; Lee e Robbins, 1995). Por outro lado, as pessoas com um nível baixo de social connectedness são incapazes de gerir as suas necessidades e sentimentos e evitam contactos sociais (Lee et al., 2001; Lee e Robbins, 1995). O social connectedness tem sido associado a várias variáveis, nomeadamente à sintomatologia psicológica. Neste sentido, vários estudos têm suportado a ideia de que o social connectedness é um fator protetor do bem-estar, estando negativamente associado à sintomatologia ansiosa e depressiva e a comportamentos autodestrutivos (Rossi et al., 2012). No mesmo sentido, os resultados do estudo de Sica et al. (2014) sugeriram que o social connectedness é uma variável mediadora da relação entre a depressão e a autoestima.

Apesar de não terem sido identificados estudos que relacionem o social connectedness e os processos de desenvolvimento da identidade propostos por Luyckx et al. (2008), os trabalhos revistos apontam para que as relações com o grupo de pares e as comparações sociais permitem consolidar as crenças sobre si próprio e sobre os outros, pelo que são essenciais ao desenvolvimento da identidade (Davidson e Youniss, 1991). Neste sentido, os resultados do estudo de Cicognani et al. (2014) com uma amostra de adolescentes mostraram que a ligação à comunidade pode afigurar-se enquanto elemento protetor dos processos de desenvolvimento identitário, na medida em que os adolescentes com um estatuto de identidade realizada apresentam níveis mais elevados de sentido de ligação à comunidade, suporte e conexão emocional com os pares e sentimento de pertença, em comparação com os adolescentes com um estatuto de identidade difuso.

\section{Adultos emergentes: tarefas desenvolvimentais}

A adultez emergente, tal como é definida por vários autores (e.g., Arnett, 2000, 2004, 2015), é uma etapa desenvolvimental que, de uma perspetiva conservadora, decorre entre os 18 e os 25 anos e é caracterizada por exploração da identidade, instabilidade, autocentração, a vivência da ambiguidade entre não ser adolescente e não se sentir adulto e a experienciação de várias possibilidades (e.g., relacionamentos amorosos, trabalho e visão do mundo). Os trabalhos realizados em Portugal, focados em adultos emergentes, apontam para que a adultez emergente possa ser definida através da maturidade psicológica e da independência económica (Mendonça et al., 2009). De acordo com Arnett $(2000,2004)$, para a maioria dos adultos emergentes, as explorações nas diversas áreas tendem a estabilizar no final da segunda década de vida, levando à formação de compromissos mais sólidos. Contudo, nos países mediterrânicos, a transição para a idade adulta 
ocorre essencialmente no interior da família, devido às dificuldades económicas e à instabilidade laboral, o que lentifica este processo (Brandão et al., 2012; Scabini et al., 2006). Apesar dos aspetos positivos da construção da identidade num contexto familiar seguro, a permanência na casa dos pais pode dificultar o processo de individuação e de autonomia (Scabini, 2000; Scabini et al., 2006), podendo também alargar, temporalmente, esta etapa desenvolvimental. Deste modo, tendo em conta o contexto socioeconómico português e os trabalhos de vários autores (Arnett, 2015; Brandão et al., 2012), neste trabalho foi alargada a faixa etária da adultez emergente para o período que decorre entre os 18 e os 30 anos de idade.

\section{O presente estudo}

Com base na literatura revista, o presente estudo pretende analisar se os valores pessoais predizem os dois ciclos de desenvolvimento da identidade e qual o papel do social connectedness enquanto mediador da relação entre os valores pessoais e os ciclos de desenvolvimento da identidade. Considerando a relação entre os valores pessoais e a identidade sugerida por vários autores (e.g., Bardi et al., 2014; Duriez et al., 2012), espera-se que os valores pessoais sejam preditores dos dois ciclos de desenvolvimento da identidade. Tendo em conta os resultados de trabalhos prévios (e.g., Gecas, 2000; Hitlin, 2003; Olsson et al., 2012), espera-se que o social connectedness se associe aos valores pessoais e aos ciclos de desenvolvimento identitário e que seja mediador da relação entre as duas variáveis. Especificamente, espera-se que a adesão a valores coletivistas e individualistas se associe positivamente ao social connectedness e, consequentemente, a níveis mais elevados de formação e de avaliação da identidade.

\section{Método}

\section{Amostra}

A amostra foi constituída por 275 participantes $(N=275)$, com idades compreendidas entre os 18 e os 30 anos $(M=22.24 ; D P=2.60)$. A maioria da amostra era do género feminino $(74,90 \%)$, vivia com a família $(55,30 \%)$ e era estudante $(71,30 \%)$, sendo que $21,50 \%$ trabalhava. Relativamente à situação afetivo-relacional, $44,70 \%$ da amostra não se encontrava numa relação amorosa, 42,10\% estava numa relação de namoro e 7,3\% coabitava/estava em união de facto. Quanto ao nível de escolaridade, $61,50 \%$ da amostra frequentava o ensino superior e $21,50 \%$ já tinha completado o ensino superior. Relativamente à zona de residência, 59,30\% da amostra residia na zona da Grande Lisboa, 18,20\% residia na zona do Centro e 10,20\% residia na Região Autónoma da Madeira. Quanto à religiosidade, 
$42,20 \%$ dos participantes eram crentes praticantes, $36,70 \%$ eram crentes não praticantes e $21,20 \%$ eram não crentes.

\section{Procedimento de recolha de dados}

A recolha de dados decorreu através de técnica de amostragem não probabilística, após a aprovação do projeto de investigação pela Comissão de Ética e Deontologia em Investigação Clínica da Escola de Psicologia e de Ciências da Vida da Universidade Lusófona de Humanidades e Tecnologias. Como critério de inclusão foram estabelecidos: idades compreendidas entre os 18 e os 30 anos; nacionalidade portuguesa.

Através do método "bola de neve", a amostra foi recolhida presencialmente, segundo procedimentos informais em grupo (e.g., contexto de sala de aula em instituições de ensino superior) ou individualmente (e.g., rede social), e on-line através da plataforma Google Docs. Com o recurso a estas estratégias, pretendeu-se uma diversificação da amostra, integrando adultos emergentes (18-30 anos) que frequentam e que não frequentam o ensino superior. Os participantes colaboraram voluntariamente e sem qualquer remuneração, após a explicitação dos objetivos do estudo e a assinatura do consentimento informado. Foram esclarecidas eventuais dúvidas relacionadas com as questões e/ou o vocabulário utilizado, quer presencialmente, quer pela disponibilização dos contactos eletrónicos da responsável pela investigação.

\section{Instrumentos}

\section{1) Questionário sociodemográfico}

Os participantes responderam a um questionário de dados pessoais e sociodemográficos. O questionário incluiu questões como idade, género, situação relacional, nível de escolaridade, profissão e religiosidade.

2) Questionário sobre Valores Pessoais Readaptado (QVPR, versão original: Schwartz, 1992; tradução e adaptação para a população portuguesa: Menezes e Campos, 1991 e Prioste et al., 2012)

O QVPR é um instrumento de autorrelato que inclui uma lista única de 63 valores. A tarefa do participante consiste em avaliar quão importante é cada valor enquanto princípio orientador da sua vida, utilizando uma escala de $0=$ nada importante a $6=$ de importância fundamental. Em cada item são apresentados um valor e uma definição, para diminuir a carga de subjetividade semântica na interpretação do item (Prioste et al., 2012). O QVPR avalia oito dimensões - tipos de valores - organizados em duas classes de valores: Individualista e Coletivista. A classe de valores Coletivista é composta pelos tipos de valores Relacional, Tradicionalismo, 
Preocupação Social e Espiritualidade e avalia a importância de valores de interdependência, i.e., que enfatizam a conexão com os outros. Inclui valores como "Família (valorização da prioridade da família no percurso de vida)" e "Generosidade (valorização de ações gratuitas em prol dos outros)". A classe de valores Individualista é composta pelos tipos de valores Aventura, Poder Social, Realização Pessoal e Equilíbrio Pessoal e avalia a importância de valores de independência em relação aos outros. Esta classe inclui valores como "Independência pessoal (valorização da autossuficiência, da autonomia)" e "Prazer (satisfação de desejos)". Quanto maior a pontuação nas classes de valores Individualista e Coletivista, maior a adesão aos valores individualistas e coletivistas. No estudo de Prioste $e t$ al. (2015), as classes Individualista e Coletivista revelaram valores adequados de consistência interna ( $\alpha=.90$ para a classe Individualista e $\alpha=.88$ para a classe Coletivista). No presente estudo, as dimensões mostraram igualmente valores de consistência interna adequados: $\alpha=.93$ para a classe dos valores Coletivistas e $\alpha=.94$ para a classe dos valores Individualistas.

3) Escala das Dimensões do Desenvolvimento Identitário (Dimensions of Identity Development Scale, DIDS; versão original: K. Luyckx et al., 2008; tradução e adaptação para a população portuguesa: Prioste et al., 2018) A DIDS é um instrumento de autorrelato que integra 25 itens e avalia o desenvolvimento da identidade através de uma escala de Likert de cinco pontos, de $1=$ discordo fortemente a 5 = concordo fortemente. A DIDS avalia cinco dimensões: Exploração em profundidade, composta por cinco itens (e.g., "Falo com outras pessoas sobre os meus planos para o futuro") que avaliam a exploração de alternativas após a adesão a compromissos; Exploração em amplitude, que integra cinco itens (e.g., "Estou a pensar em diferentes estilos de vida que podem ser bons para mim") que medem a exploração de alternativas prévia à adesão a compromissos; Compromisso, que inclui cinco itens (e.g., "Tenho uma imagem sobre o que vou fazer no futuro") que avaliam a adesão a compromissos; Identificação com o compromisso, constituída por cinco itens (e.g., "Os meus planos para o futuro dão-me autoconfiança") que avaliam a segurança e identificação em relação aos compromissos; e Exploração ruminativa, composta por cinco itens (e.g., "Tenho dúvidas sobre o que quero realmente alcançar na vida") que avaliam a exploração progressiva de diversas alternativas e a não adesão a compromissos.

No estudo de validação da DIDS (Luyckx et al., 2008), com uma amostra de jovens adultos, as dimensões da escala mostraram níveis adequados de consistência interna, variando entre $\alpha=.79$ para a dimensão Exploração em profundidade e entre $\alpha=.86$ para as dimensões Compromisso, Identificação com 
o compromisso, Exploração em amplitude e Exploração ruminativa. No presente estudo, as dimensões mostraram igualmente valores de consistência interna adequados: $\alpha=.88$ para a dimensão Compromisso; $\alpha=.80$ para a dimensão Identificação com compromisso; na dimensão Exploração em amplitude $\alpha=.76$; na dimensão Exploração em profundidade $\alpha=.89$; e $\alpha=.68$ na dimensão Exploração ruminativa.

4) Social Connectedness Scale - Revised (SCS-R; versão original: Lee et al., 2001; tradução e adaptação para a população portuguesa: Francisco et al., 2011)

A SCS-R é um instrumento de autorrelato que avalia a proximidade interpessoal que uma pessoa experiencia nas suas relações com o mundo social (Lee et al., 2001) através de uma escala de Likert de seis pontos, de $1=$ discordo fortemente a 6 = concordo fortemente. A escala é composta por 20 itens, sendo que dez dos itens estão enunciados negativamente (e.g., "Mesmo entre pessoas que conheço, não sinto que realmente pertença ali") e os restantes dez estão formulados na positiva (e.g., "Vejo as pessoas como amigáveis e acessíveis"). Quanto maior a pontuação na escala, maior o nível de social connectedness.

No estudo de Malaquias et al. (2015), foram também encontrados valores adequados de consistência interna $(\alpha=.90)$. No presente estudo, a escala revelou igualmente um bom nível de consistência interna $(\alpha=.92)$.

\section{Procedimento de análise de dados}

Inicialmente, foi realizada a análise descritiva dos dados e foram analisadas as correlações entre as variáveis social connectedness, valores coletivistas, valores individualistas, formação da identidade e avaliação da identidade através do software Statistical Package for the Social Science 22. De seguida, o modelo conceptual proposto foi testado através da Análise de Equações Estruturais (Structural Equation Modeling, SEM), usando o método da máxima verosimilhança, com recurso ao software AMOS 22 (Arbuckle, 2012).

O modelo proposto (Figura 1), que incluía sete variáveis latentes, testou o papel do social connectedness como variável mediadora da relação entre os valores e os ciclos de desenvolvimento identitário. No modelo, a variável independente (valores pessoais) foi especificada como uma variável latente de segunda ordem e foi medida através dos valores individualistas e dos valores coletivistas; a variável dependente (ciclos de desenvolvimento identitário) foi também especificada como uma variável latente de segunda ordem e foi medida através da formação de compromisso (FC) e da avaliação de compromisso (AC). No sentido de simplificar o modelo e de reduzir o número de parâmetros a serem estimados, foram usadas parcelas de 
multi-item para especificar as variáveis (Little et al., 2002). Para garantir a identificação estatística do modelo, a carga fatorial de uma das parcelas de cada variável latente foi constrangida a 1.00 .

A avaliação do ajustamento do modelo aos dados foi realizada com base na análise dos seguintes índices de ajustamento: qui-quadrado $\left(\chi^{2}\right)$, comparative fit index (CFI) e root-mean-square error of approximation (RMSEA). De acordo com Garson (2012), valores de CFI $\geq$ a .90, e valores de RMSEA $\leq 0.06$ são indicativos de um bom ajustamento do modelo aos dados.

\section{Resultados}

\section{Estatística descritiva e análise de correlações}

A estatística descritiva e as correlações entre as variáveis encontram-se apresentadas no Quadro 1. Os valores coletivistas apresentam-se positivamente correlacionados com os valores individualistas, o social connectedness, a formação e a avaliação do compromisso. Observam-se correlações positivas entre os valores individualistas e o social connectedness e a formação do compromisso. O social connectedness encontra-se positivamente correlacionado com a avaliação e a formação do compromisso. Por último, a formação do compromisso encontra-se positivamente correlacionada com a avaliação do compromisso.

QUADRO 1 - Estatística descritiva e correlação entre valores, social connectedness e ciclos identitários $(\mathrm{N}=275)$

\begin{tabular}{lccccc}
\hline \multicolumn{1}{c}{ Variáveis } & 1 & 2 & 3 & 4 & 5 \\
\hline 1. Valores coletivistas & - & & & & \\
2. Valores individualistas & $.85^{* *}$ & - & & & \\
3. Social connectedness & $.27^{* *}$ & $.23^{* *}$ & - & & \\
4. Formação de compromisso & $.12^{* *}$ & $.14^{* *}$ & $.34^{* *}$ & - & \\
5. Avaliação de compromisso & $.14^{*}$ & .11 & $.29^{* *}$ & $.60^{* *}$ & - \\
\hline$M$ & 4.68 & 4.59 & 4.58 & 3.92 & 3.67 \\
\multicolumn{1}{c}{ DP } & .69 & .63 & .76 & .48 & .56 \\
\hline
\end{tabular}

Nota: ${ }^{* *} p<.01 ;{ }^{*} p<.05$

\section{Papel mediador do social connectedness}

Inicialmente, foi analisada a variável valores pessoais como preditor dos ciclos de desenvolvimento da identidade. Os parâmetros estimados mostraram que os valores pessoais estão significativamente associados à variável dependente $(\beta=.18, p<.001)$, mas os índices de ajustamento revelam que o modelo não é adequado aos dados $(\chi 2(1, \mathrm{~N}=275)=2.04$, n.s., 
$\mathrm{CFI}=.98, \mathrm{RMSEA}=.06$. Posteriormente, foi testada a hipótese de que o efeito do preditor dos valores nos ciclos de desenvolvimento da identidade seria mediado pelo social connectedness (Figura 1). Os índices de ajustamento do modelo revelam que o modelo conceptual proposto é adequado aos dados: $\chi 2(11, N=275)=20.51, p<.05$, CFI $=.99$, RMSEA $=.056$, explicando $18 \%$ da variância. Os resultados indicam um efeito de mediação do social connectedness na relação entre os valores e os ciclos de desenvolvimento da identidade (Sobel $=2.30, p<.005)$.

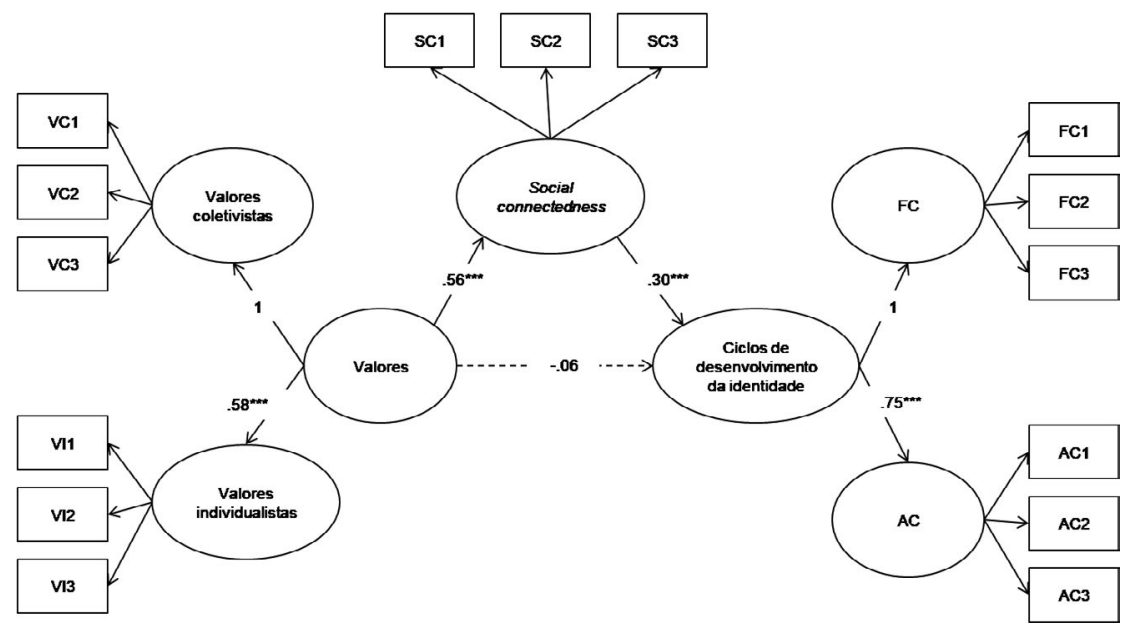

FIGURA 1 - Coeficientes estandardizados do modelo de mediação proposto, da relação entre os valores pessoais, o social connectedness e os ciclos de desenvolvimento da identidade

Nota: VC1-VC3 = parcelas relativas aos valores coletivistas da escala QVP-R; VI1-3C3 = parcelas relativas aos valores individualistas da escala QVP-R; SC1-SC3 = parcelas relativas à escala SCS-R; FC = formação do compromisso; FC1-FC3 = parcelas relativas à escala $\mathrm{FC} ; \mathrm{AC}=$ avaliação do compromisso; $\mathrm{AC1}-\mathrm{AC} 1=$ parcelas relativas à escala $\mathrm{AC}\left({ }^{*} p<.05 ;{ }^{* *} p<.01 ;{ }^{* * *}\right.$ $p<.001$ ).

\section{Discussão}

O presente estudo pretendeu investigar: (1) a relação entre os valores coletivistas e individualistas, os ciclos de identidade propostos por Luyckx et al. (2008) e o social connectedness; (2) se os valores pessoais predizem os ciclos de desenvolvimento da identidade; e (3) o papel do social connectedness enquanto mediador da relação entre os valores pessoais e os ciclos de desenvolvimento da identidade. Este trabalho tem como finalidade contribuir para o enriquecimento do conhecimento científico sobre o impacto 
de variáveis individuais e relacionais no desenvolvimento da identidade em adultos emergentes portugueses. Para além disso, pretendeu-se colmatar algumas das lacunas identificadas na literatura, nomeadamente a inexistência de estudos que relacionam os valores pessoais e os ciclos de desenvolvimento da identidade tendo por base o modelo de Luyckx et al. (2008).

Apesar de os índices de ajustamento do modelo de predição terem revelado fragilidades, os resultados obtidos apontam para que os valores pessoais sejam preditores dos ciclos de desenvolvimento da identidade, i.e., quanto maior for a adesão dos adultos emergentes aos valores colectivistas e individualistas, maior será o nível de formação e de avaliação dos compromissos. Estes resultados apoiam os trabalhos que sugerem que a adesão a valores pessoais influencia o desenvolvimento da identidade (Hitlin, 2003). Por outro lado, tal como sugeriram Bardi et al. (2014), as associações positivas observadas entre a formação do compromisso e os valores individualistas e coletivistas realçam a importância dos objetivos pessoais e das normas sociais na exploração de alternativas e projetos de vida e no estabelecimento do compromisso com esses projetos e essas alternativas.

Os resultados obtidos através da análise das correlações apontam para que a avaliação do compromisso se correlacione com os valores coletivistas e não com os valores individualistas, sugerindo que a adesão a valores que privilegiem o coletivo/grupo é essencial para que os adultos emergentes se identifiquem com os seus compromissos e os explorem ativa e profundamente. Deste modo, os resultados suportam a literatura que realça a importância da conexão com o grupo de pares e das comparações sociais na consolidação das crenças individuais e no desenvolvimento da identidade (Davidson e Youniss, 1991). O facto de os nossos resultados apontarem para que a avaliação do compromisso se associe aos valores coletivistas vai ao encontro de alguns estudos realizados em Portugal (e.g., Prioste et al., 2017; Ramos, 2006) que sugerem que os valores da interdependência são, tendencialmente, mais valorizados em Portugal, constituindo-se como princípios orientadores da sociedade portuguesa. Neste sentido, este resultado também poderá ser interpretado atendendo à perspetiva das fontes de valores (Pereira et al., 2005) que sugere que os valores, além de expressarem necessidades e motivações individuais, representam princípios relativos à forma como a sociedade se deve organizar, estando, por isso, entretecidos nas identidades sociais (Estramiana et al., 2013). A hipótese referente ao papel mediador do social connectedness na relação entre os valores pessoais e os ciclos de desenvolvimento da identidade foi apoiada pelos resultados obtidos. Estes resultados revelam que os adultos emergentes 
que aderem a valores individualistas e coletivistas, experienciam as suas relações interpessoais como próximas e, consequentemente, apresentam níveis superiores de compromisso com os estilos de vida experienciados após várias alternativas, continuando a explorá-los. Assim, os resultados obtidos, quer através das correlações, quer através do teste do modelo proposto, corroboram conclusões de trabalhos anteriores, mostrando que os valores pessoais preservam o sentido de ligação com o mundo social, diminuindo a perceção de desconexão com os outros e favorecendo a formação e a avaliação de compromissos (Cicognani et al., 2014). O sentimento de proximidade interpessoal poderá, assim, funcionar como "o padrão que liga" (Bateson, 1987) os princípios orientadores da vida dos adultos emergentes à exploração e identificação das escolhas, através da perceção de que o self é significativo e relevante para os outros (Adams e Marshall, 1996). Neste sentido, as relações interpessoais e sociais poderão funcionar, por um lado, como um meio privilegiado para experienciar os valores pessoais e, por outro lado, como um contexto de validação da identidade pessoal e das escolhas assumidas ou exploradas (Davidson e Youniss, 1991).

Estes resultados suportam também a ideia de que a desvalorização de valores individualistas e coletivistas se associa à indisponibilidade ou incapacidade relacional e social e, consequentemente, à incapacidade para formar e avaliar compromissos identitários. Deste modo, os adultos emergentes que têm dificuldades na adesão a princípios orientadores da vida, não se conseguem sentir sintonizados e envolvidos com os outros, pelo que tendem a apresentar dificuldades no estabelecimento de compromissos em relação a projetos ou opções de vida.

\section{Implicações para a literatura e para a prática clínica}

Este trabalho poderá ser um contributo no mapeamento da relação entre os valores pessoais e os ciclos de desenvolvimento da identidade propostos por Luyckx et al. (2008), através do estudo do papel do social connectedness enquanto mediador. Desta forma, os resultados obtidos poderão ter implicações para a literatura na área da adultez emergente ao revelarem que, nesta etapa desenvolvimental, a adesão a valores individualistas e coletivistas contribui para a formação e avaliação de compromissos identitários. Este trabalho pode ainda apresentar implicações para a literatura na área da identidade e dos valores pessoais ao mostrar que o social connectedness é um mecanismo explicativo da relação entre os valores e o desenvolvimento da identidade, i.e., o sentido de ligação com os outros e com o mundo poderá funcionar como um contexto de validação dos valores e de desenvolvimento da identidade. 
As variáveis analisadas neste trabalho sublinham também a relação dialética entre o nível individual (i.e., foco nos valores individualistas e na identidade) e o nível relacional, especificamente, a necessidade de manutenção de relações significativas e próximas com os outros. Consistente com a perspetiva sistémica, o estudo enfatiza a interindependência destes níveis na construção do self dos adultos emergentes: os contextos relacionais e o sentimento de valorização dos outros são essenciais para a individuação, mas a diferenciação dos outros também é fulcral para a autonomização (Adams e Marshall, 1996). Os resultados deste estudo também poderão contribuir para a prática clínica, ao sugerirem que, na adultez emergente, o desenvolvimento da identidade está associado à adesão a valores coletivistas e individualistas, bem como a níveis elevados de social connectedness. Neste sentido, as conclusões deste trabalho podem, potencialmente, contribuir para a definição de linhas orientadoras no desenvolvimento e implementação de intervenções individuais específicas focadas no desenvolvimento da identidade em adultos emergentes, através do foco nos valores a que os adultos emergentes aderem, na importância que atribuem a cada valor e no impacto da adesão a valores específicos na pertença e na proximidade interpessoal com diferentes grupos sociais. Considerando a relevância que a exploração da identidade nas áreas relacional e laboral assume no decorrer da adultez emergente, os resultados poderão sublinhar ainda a importância dos valores coletivistas (e.g., família, amizade, amor, respeito pelos outros) e do social connectedness na exploração da identidade na área relacional e dos valores individualistas (e.g., trabalho, sucesso, ambição, escolha de objetivos de vida) na exploração da identidade na área laboral.

\section{Limitações e estudos futuros}

Embora o presente estudo possa contribuir para o aprofundamento do conhecimento sobre o impacto de variáveis individuais e relacionais no desenvolvimento da identidade em adultos emergentes portugueses, apresenta algumas lacunas. $O$ facto de a amostra utilizada neste estudo ser não probabilística não permite a generalização dos resultados à população de adultos emergentes portugueses. A amostra é discrepante em relação ao género e à ocupação, sendo maioritariamente constituída por estudantes universitários do género feminino. Em relação aos instrumentos, o facto de terem sido utilizados instrumentos de autorrelato levanta questões em relação aos enviesamentos originados pela desiderabilidade social. Para além disso, o facto de o estudo ter um desenho transversal, não possibilita o estabelecimento de relações de causalidade entre as variáveis em estudo. Por último, o facto de não ter sido incluído o processo de exploração 
ruminativa não nos permite estudar mais detalhadamente a dinâmica do desenvolvimento da identidade proposta por Luyckx et al. (2008).

Com o intuito de colmatar estas lacunas, seria importante analisar os resultados obtidos em estudos futuros com uma amostra de adultos emergentes mais heterogénea que incluísse uma percentagem maior de participantes do género masculino e de adultos emergentes trabalhadores. Seria também interessante incluir adultos emergentes "nem-nem", i.e., que não trabalham e não estudam.

Alguns trabalhos têm demonstrado o papel do género, da psicopatologia e da parentalidade helicóptero nos valores pessoais e/ou no desenvolvimento da identidade em adultos emergentes (e.g., Luyckx et al., 2013; Padilla-Walker e Nelson, 2012; Torres e Brites, 2006). No sentido de aumentar a percentagem de variância explicada, os estudos futuros poderiam ser desenvolvidos no sentido de compreender o papel moderador do género nas relações analisadas e a associação entre a parentalidade helicóptero, a psicopatologia, o desenvolvimento da identidade, os valores pessoais e o social connectedness.

Revisto por Sofia Silva

\section{Referências bibliográficas}

Adams, Gerald; Marshall, Sheila (1996), "A Developmental Social Psychology of Identity: Understanding the Person-in-Context", Journal of Adolescence, 19(5), 429-442. Arbuckle, James (2012), Amos 21.0 User's Guide. Chicago: SPSS-IBM.

Arnett, Jeffrey Jensen (2000), "Emerging Adulthood: A Theory of Development from the Late Teens through the Twenties", American Psychologist, 55(5), 469-480.

Arnett, Jeffrey Jensen (2004), Emerging Adulthood: The Winding Road from the Late Teens through the Twenties. New York: Oxford University Press.

Arnett, Jeffrey Jensen (2015), Emerging Adulthood: The Winding Road from the Late Teens through the Twenties. New York: Oxford University Press [2." ed.].

Bardi, Anat; Jaspal, Rusi; Polek, Ela; Schwartz Shalom (2014), "Values and Identity Process Theory (IPT): Theoretical Integration and Empirical Interactions", in Rusi Jaspal; Glynis M. Breakwell (orgs.), Identity Process Theory: Identity, Social Action and Social Change. New York: Cambridge University Press.

Bateson, Gregory (1987), Natureza e espírito. Lisboa: Dom Quixote. Tradução de Maria do Rosário Carrilho.

Brandão, Tânia; Saraiva, Luísa; Matos, Paula Mena (2012), "O prolongamento da transição para a idade adulta e o conceito de adultez emergente: especificidades do contexto português e brasileiro", Análise Psicológica, 3, 301-313. 
Caprara, Gian Vittorio; Schwartz, Shalom; Capanna, Cristina; Vecchione, Michele; Barbaranelli, Claudio (2006), "Personality and Political: Values, Traits, and Political Choice”, Political Psychology, 27(1), 1-28.

Cicognani, Elvira; Klimstra, Theo; Goossens, Luc (2014), "Sense of Community, Identity Statuses, and Loneliness in Adolescence: A Cross-National Study on Italian and Belgian Youth”, Journal of Community Psychology, 42(4), 414-432.

Cordeiro, Pedro Miguel Gomes; Paixão, Maria Paula; Lens, Willy; Lacante, Marlies; Luyckx, Koen (2015), "Cognitive-Motivational Antecedents of Career Decision-Making Processes in Portuguese High School Students: A Longitudinal Study", Journal of Vocational Bebavior, 90, 145-153.

Davidson, Philip; Youniss, James (1991), “Which Comes First, Morality or Identity?”, in William Kurtines; Jacob Gewirtz; Jacob Lamb (orgs.), Handbook of Moral Behavior and Development: Theory, Research and Applications. New Jersey: Erlbaum.

Duriez, Bart; Luyckx, Koen; Soenens, Bart; Berzonsky, Michael (2012), “A Process-Content Approach to Adolescent Identity Formation: Examining Longitudinal Associations between Identity Styles and Goal Pursuits", Journal of Personality, 80(1), 135-161.

Erikson, Erik (1968), Identity: Youth and Crisis. New York: Norton.

Estramiana, José Luis; Pereira, Cícero; Monter, Miryam Rodríguez; Zlobina, Anna (2013), "Valores sociais", in Leoncio Camino; Ana Raquel Rosas Torres; Marcus Eugénio Oliveira Lima; Marcos Emanoel Pereira (orgs.), Psicologia social: temas e teorias. Brasília: TechnoPolitik, 311-355 [2. ${ }^{a}$ ed.].

Gecas, Viktor (2000), "Values Identities, Self-Motives, and Social Movements", in Sheldon Strycker; Timothy Owens; Robert White (orgs.), Self, Identity, and Social Movements. Minneapolis: University of Minnesota Press, 93-109.

Grotevant, Harold (1987), "Toward a Process Model of Identity Formation”, Journal of Adolescent Research, 2(3), 203-222.

Hermans, Hubert; Oles, Piotr (1993), "The Personal Meaning of Values in a Rapid Changing Society”, The Journal of Social Psychology, 134(5), 569-579.

Hitlin, Steven (2003), "Values as the Core of Personal Identity: Drawing Links between Two Theories of the Self", Social Psychology Quarterly, 66(2), 118-137.

Karás, Dominika; Cieciuch, Jan; Negru, Oana; Crocetti, Elisabetta (2014), "Relationships between Identity and Well-Being in Italian, Polish, and Romanian Emerging Adults", Social Indicators Research, 121, 727-743.

Lee, Richard; Draper, Matthew; Lee, Sujin (2001), "Social Connectedness, Dysfunctional Interpersonal Behaviors, and Psychological Distress: Testing a Mediator Model”, Journal of Counseling Psychology, 48(3), 310-318.

Lee, Richard; Robbins, Steven Bernard (1995), "Measuring Belongingness: The Social Connectedness and the Social Assurance Scales”, Journal of Counseling Psychology, 42(2), 232-241. 
Lee, Richard; Robbins, Steven Bernard (1998), “The Relationship between Social Connectedness and Anxiety, Self-Esteem, and Social Identity”, Journal of Counseling Psychology, 45(3), 338-345.

Little, Todd; Cunningham, William; Shahar, Golan; Widaman, Keith (2002), "To Parcel or Not to Parcel: Exploring the Question and Weighing the Merits", Structural Equation Modeling, 9, 151-173.

Luyckx, Koen; Goossens, Luc; Soenens, Bart (2006), “A Developmental-Contextual Perspective on Identity Construction in Emerging Adulthood: Change Dynamics in Commitment Formation and Commitment Evaluation", Developmental Psychology, 42(2), 366-380.

Luyckx, Koen; Klimstra, Theo; Duriez, Bart; Petegem, Stijn Van; Beyers, Wim (2013), "Personal Identity Processes from Adolescence through the Late 20s: Age Trends, Functionality, and Depressive Symptoms”, Social Development, 22(4), 701-721. DOI: $10.1111 /$ sode. 12027

Luyckx, Koen; Schwartz, Seth; Berzonsky, Michael; Soenens, Bart; Vansteenkiste, Maarten; Smits, Ilse; Goossens, Luc (2008), “Capturing Ruminative Exploration: Extending the Four-Dimensional Model of Identity Formation in Late Adolescence", Journal of Research in Personality, 42(1), 58-82. DOI: 10.1016/j.jrp.2007. 04.004

Luyckx, Koen; Schwartz, Seth; Goossens, Luc; Beyers, Wim; Missotten, Lies (2011), "Processes of Personal Identity Formation and Evaluation", in Seth Schwartz; Koen Luyckx; Vivian Vignoles (orgs.), Handbook of Identity Theory and Research. New York: Springer, 77-98.

Marcia, James (2002), "Identity and Psychosocial Development in Adulthood", Identity: An International Journal of Theory and Research, 2(1), 7-28.

Mendonça, Marina; Andrade, Cláudia; Fontaine, Anne Marie (2009), “Transição para a idade adulta e adultez emergente: adaptação do Questionário de Marcadores da Adultez junto de jovens portugueses", Psychologica, 51, 147-168.

Menezes, Isabel; Campos, Bártolo Paiva (1991), "Estrutura dos Valores: Estudo Transversal", Psychologica, 6, 129-147.

Olsson, Craig; McGee, Rob; Nada-Raja, Shyamala; Williams, Sheila (2012), “A 32 Year Longitudinal Study of Child and Adolescent Pathways to Well-Being in Adulthood”, Journal of Happiness Studies, 14(3), 1069-1083.

Padilla-Walker, Laura; Nelson, Larry (2012), “Black Hawk Down?: Establishing Helicopter Parenting as a Distinct Construct from other Forms of Parental Control During Emerging Adulthood”, Journal of Adolescence, 35(5), 1177-1190. DOI: 10.1016/j.adolescence.2012.03.007

Pereira, Cícero; Camino, Leoncio; Costa, Joselí Bastos (2005), "Um estudo sobre a integração dos níveis de análise dos sistemas de valores”, Psicologia: Reflexão e Crítica, 18(1), 16-25. 
Prince-Gibson, Eetta; Schwartz, Shalom (1998), "Value Priorities and Gender", Social Psychology Quarterly, 61(1), 49-67.

Prioste, Ana; Narciso, Isabel; Gonçalves, Miguel (2012), "Questionário de valores pessoais readaptado: processo de desenvolvimento e contributos iniciais para a validação", Revista Iberoamericana de Diagnóstico y Evaluatión Psicológica, 1(34), 175-199.

Prioste, Ana; Narciso, Isabel; Gonçalves, Miguel; Pereira, Cícero Roberto (2015), "Family Relationships and Parenting Practices: A Pathway to Adolescents' Collectivist and Individualist Values?”, Journal of Child and Family Studies, 24(11), 3258-3267.

Prioste, Ana; Narciso, Isabel; Gonçalves, Miguel; Pereira, Cícero Roberto (2017), "Values' Family Flow: Associations between Grandparents, Parents and Adolescent Children”, Journal of Family Studies, 23(1), 98-117.

Prioste, Ana; Lugar, Ana; Paulino, Paula; Jongenelen, Inês; Rosa, Pedro Joel (2018), "Escala das Dimensões do Desenvolvimento da Identidade: Estudos Psicométricos Iniciais”, Psicologia, 32(2), 1-14. DOI: 10.17575/rpsicol.v32i2.1244

Ramos, Alice (2006), "Social Values Dynamics and Socio-economic Development", Portuguese Journal of Social Science, 5, 35-64. DOI:10.1386/pjss.5.1.35/1

Ritchie, Rachel; Meca, Alan; Madrazo, Vanessa; Schwartz, Seth; Hardy, Sam; Zamboanga, Byron; Weisskirch, Robert; Kim, Su Yeon; Whitbourne, Susan Krauss; Ham, Linsay; Lee, Richard (2013), "Identity Dimensions and Related Processes in Emerging Adulthood: Helpful or Harmful?, Journal of Clinical Psychology, 69(4), 415-432. DOI: $10.1002 /$ jclp.21960

Rossi, Alessandro; Stratta, Paolo; Capanna, Cristina (2012), "Social Connectedness and Psychopathology”, Journal of Psychopathology, 18(4), 305-308.

Scabini, Eugenia (2000), "Parent-Child Relationships in Italian Families: Connectedness and Autonomy in the Transition to Adulthood", Psicologia: Teoria e Pesquisa, 16(1), 23-30.

Scabini, Eugenia; Marta, Elena; Lanz, Margherita (2006), The Transition to Adulthood and Family Relations: An Intergenerational Perspective. London: Psychology Press.

Schwartz, Shalom (1992), "Universals in the Content and Structure of Values: Theory and Empirical Tests in 20 Countries", in Mark Zanna (org.), Advances in Experimental Social Psychology. New York: Academic Press, 1-65.

Schwartz, Shalom; Bilsky, Wolfgang (1990), "Toward a Theory of the Universal Content and Structure of Values: Extensions and Cross Cultural Replications", Journal of Personality and Social Psychology, 58(5), 878-891.

Schwartz, Seth; Beyers, Wim; Luyckx, Koen; Soenens, Bart; Zamboanga, Byron; Forthun, Larry; Hardy, Sam; Vazsonyi, Alexander; Ham, Lindsay; Kim, Su Yeon; Whitbourne, Susan Krauss; Waterman, Alan (2011), "Examining the Light and Dark 
98 | Ana Prioste, Cristina Ascenção, Eunice Magalhães, Inês Jongenenlen

Sides of Emerging Adults' Identity: A Study of Identity Status Differences in Positive and Negative Psychosocial Functioning”, Journal of Youth and Adolescence, 40(7), 839-859. DOI: $10.1007 /$ s10964-010-9606-6

Schwartz, Shalom; Cieciuch, Jan; Vecchione, Michele; Davidov, Eldad; Fischer, Ronald; Beieilein, Constanze; Ramos, Alice; Verkasalo, Markku; Lonnqvist, Jan-Erik; Demirutki, Kursad; Dirilen-Gumus, Ozlem; Konty, Mark (2012), "Redefining the Theory of Basic Individual Values", Journal of Personality and Social Psychology, 103, 663-688. DOI: 10.1037/a0029393

Seligman, Clive; Katz, Albert (1996), "The Dynamics of Value Systems”, in Clive Seligman; James Olson; Mark Zanna (orgs.), The Psychology of Values: The Ontario Symposium, vol. 8. Mahwah, NJ: LEA, 53-75.

Sica, Luigia Simona; Sestito, Laura Aleni; Ragozini, Giancarlo (2014), "Identity

Coping in the First Years of University: Identity Diffusion, Adjustment and Identity Distress", Journal of Adult Development, 22, 159-172. DOI: 10.1007/ s10804-014-9188-8

Torres, Anália; Brites, Rui (2006), "European Attitudes and Values: The Perspective of Gender in a Transverse Analysis”, Portuguese Journal of Social Science, 5(3), 179-214.

Artigo recebido a 01.09.2017

Aprovado para publicação a 17.07.2018

\section{Ana Prioste}

Escola de Psicologia e Ciências da Vida, HeiLab, Universidade Lusófona de Humanidades e Tecnologias | Faculdade de Psicologia, Universidade de Lisboa ULHT, Campo Grande, 376, 1749-024 Lisboa, Portugal Contacto: ana.prioste@ulusofona.pt ORCID: https://orcid.org/0000-0001-8881-8963

\section{Cristina Ascensão}

Escola de Psicologia e Ciências da Vida, Universidade Lusófona de Humanidades e Tecnologias Campo Grande, 376, 1749-024 Lisboa, Portugal

Contacto: cristinaascensao89@gmail.com

\section{Eunice Magalhães}

Escola de Psicologia e Ciências da Vida, Universidade Lusófona de Humanidades e Tecnologias | Instituto Universitário de Lisboa (ISCTE-IUL), CIS-IUL

ULHT, Campo Grande, 376, 1749-024 Lisboa, Portugal

Contacto: eunice_magalhaes@iscte-iul.pt

ORCID: https://orcid.org/0000-0002-1421-0397 


\section{Inês Jongenelen}

Escola de Psicologia e Ciências da Vida, HeiLab, Universidade Lusófona de Humanidades e Tecnologias Campo Grande, 376, 1749-024 Lisboa, Portugal

Contacto: ijongenelen@ulp.pt

ORCID: https://orcid.org/0000-0002-4478-8739

\section{The Relationship between Personal Values, Social Connectedness and Identity Development of Portuguese Emerging Adults}

Personal values and social connectedness influence the development of identity. Through a quantitative design and a sample of 275 emerging adults, the present study sought to analyze: the relationship between personal collectivist and individualist values, the two cycles of identity development (the formation and evaluation of commitments) and social connectedness; whether personal values may predict the cycles of one's identity development; and the mediating role of social connectedness in the relationship between personal values and the cycles of identity development. Results showed that social connectedness mediated the relationship between personal values and the cycles of identity development, suggesting that a sense of interpersonal closeness can act as an endorsement mechanism for personal values and that it may contribute to the development of identity. This study has implications for the literature related to the development of identity in emerging adulthood.

Keywords: emerging adults; individual development; personal identity; Portugal; social interaction.

\section{Rapport entre les valeurs person- nelles, le social connectedness et le développement de l'identité chez les adultes portugais émergents}

Les valeurs personnelles et le social connectedness (les liens sociaux) contribuent au développement de l'identité. Grâce à un dessin quantitatif transversal et à un échantillon de 275 adultes émergents, la présente étude visait à analyser: la relation entre les valeurs personnelles collectivistes et individualistes, les deux cycles du développement de l'identité (formation et évaluation de l'engagement) et le social connectedness; si les valeurs personnelles sont des prédicteurs des cycles de développement de l'identité; et le rôle médiateur du social connectedness dans le rapport entre les valeurs personnelles et les cycles de développement de l'identité. Les résultats montrent que le social connectedness jouent un rôle de médiateur dans le rapport entre les valeurs personnelles et les cycles de développement de l'identité, suggérant que le sentiment de proximité interpersonnelle fonctionne comme un mécanisme de confirmation des valeurs personnelles et contribue au développement de l'identité. Cette étude a des répercussions sur la littérature ayant trait au développement de l'identité à l'âge adulte émergent.

Mots-clés: adultes émergents; développement individuel; identité personnelle; interaction sociale; Portugal. 
